\title{
The choose-short effect in pigeon memory for stimulus duration: Subjective shortening versus coding models
}

\author{
PHILIPP J. KRAEMER, DWIGHT S. MAZMANIAN, and WILLIAM A. ROBERTS \\ University of Western Ontario, London, Ontario, Canada
}

\begin{abstract}
A symbolic delayed matching procedure may be used to study memory for stimulus duration in pigeons. Short and long presentations of a light sample stimulus are mapped onto the choice of visually differentiated comparison keys. When delay is varied in such a symbolic delayed matching procedure, pigeons show increasing preference for the short-sample key as the delay becomes longer (choose-short effect), even after a long sample stimulus has been presented. Two theoretical explanations of the choose-short effect are suggested. A subjective shortening model holds that the choose-short effect arises from progressive shortening of the memory of stimulus duration as the delay proceeds. An alternative coding model suggests that the choose-short effect arises from stimulus generalization after an initial response instruction to peck the long-sample key has been forgotten. These two models were tested by training pigeons to peck a third comparison key after no sample stimulus had been presented. Shifts in key preferences over delays ranging from 0 to $21 \mathrm{sec}$ clearly supported the coding model.
\end{abstract}

In recent articles on short-term memory for stimulus duration, a choose-short effect has been found with both rats (Church, 1980; S. Roberts, 1982) and pigeons (Spetch \& Wilkie, 1982, 1983). For example, Church required rats to press one lever if a 2 -sec (short) signal had been presented and another lever if an 8-sec (long) signal had been presented. When the retention interval between the time signal and the opportunity to press a lever was increased to $32 \mathrm{sec}$, accuracy was low and animals showed a preference for the lever associated with the 2-sec stimulus (choose-short effect). In a further experiment, Church extended the retention interval to $8 \mathrm{sec}$ and varied the duration of the time signal over a number of values. The function relating choice of the long response to signal duration tended to flatten at the 8-sec retention interval, but the point of subjective equality (PSE) did not change as the retention interval increased to $8 \mathrm{sec}$. Church argued that had the memory of a time duration gradually shortened through the resetting of an internal clock over the retention interval, the PSE should have increased with the retention interval. He concluded that rats forgot time duration on a nontime dimension and that the choose-short effect represented a guessing bias.

In similar experiments with pigeons, Spetch and Wilkie trained birds to perform a delayed symbolic matching task in which pecking one comparison key was rewarded following a 2-sec sample stimulus and pecking a second comparison key was rewarded following a 10-sec sample

Support for this research was provided by Grant A7894 from the Natural Sciences and Engineering Research Council of Canada. Requests for reprints should be sent to William A. Roberts, Department of Psychology, University of Western Ontario, London, Ontario, Canada N6A $5 \mathrm{C} 2$. stimulus. As the delay following the sample stimulus was extended, pigeons showed better retention of the short stimulus than of the long stimulus. In other words, pigeons showed a preference for the comparison key mapped onto the short sample stimulus following both short and long sample stimuli. In an additional experiment, Spetch and Wilkie (1983) showed that psychophysical functions relating accuracy to sample duration tended to flatten as delay increased, and that the PSE was significantly higher at a delay of $20 \mathrm{sec}$ than at delays of 0 and $5 \mathrm{sec}$. They argued that these data pointed toward a subjective shortening or gradual diminution of the memory of sample stimulus duration during the retention interval.

Two models of retention of stimulus duration are considered here. In keeping with Church's ideas, one model suggests that information presented to animals on a time dimension is coded into categorical information on some other dimension. This possibility can be considered in terms of current notions of animals' remembering retrospectively or prospectively (Honig \& Thompson, 1982). Within a retrospective framework, animals might code samples of varying durations into one of two categories, short or long, and remember only the category. Alternatively, Honig (1981) has suggested, from a prospective point of view, that animals might form a response decision immediately after the time signal has been presented and then increasingly forget the response decision over the retention interval. Within Spetch and Wilkie's model, on the other hand, temporal information is not coded into another dimension but is remembered as the specific time duration presented. However, the time duration or clock setting remembered is modified over the retention interval, either by a process similar to trace decay (W. A. Roberts \& Grant, 1976) or by the gradual 
resetting of an internal clock (Church, 1980). Both the coding model and the Spetch and Wilkie model assume a continuous interaction between working and reference memory (Honig, 1978). In two-dimensional or coding models, rules stored in reference memory dictate the coding of different signal durations into categorical codes and the relationships between those codes and response choice. In the one-dimensional or subjective-shortening model, representations of short- and long-sample durations established in training are held in reference memory, and the similarity of the duration residing in working memory to the reference memory standard durations determines the response choice (Spetch \& Wilkie, 1983).

The observation of a choose-short effect follows in a straightforward manner from the notion of subjective shortening. As memory of a long sample stimulus gradually shortens, it will become progressively more similar to a short sample stimulus and result in responses to the short-sample key. For a coding model, however, it is not so obvious why animals would be biased toward the shortsample key after forgetting the long-sample category or response instruction. One possible explanation for the choose-short effect is to assume that animals form a response instruction based on the similarity of the existing stimulus duration to durations held in reference memory. If the categorical information stored on a long sample stimulus trial has been lost from working memory, the existing stimulus duration is $0 \mathrm{sec}$. If reference memory is now searched for the most appropriate response rule, the response category chosen is more likely to be the one that is appropriate for a short, or 2-sec, sample than it is to be the one appropriate for a long, or 10sec, sample, since $0 \mathrm{sec}$ is more similar to $2 \mathrm{sec}$ than to $10 \mathrm{sec}$ on a temporal dimension. Therefore, stimulus generalization can be used to explain the choose-short effect with both coding and subjective-shortening models. The notion that the absence of a sample duration may be coded into a respond-short instruction is supported by the observation made with both rats and pigeons that animals show a preference for the short response when no signal duration has been presented (Church, 1980; Spetch \& Wilkie, 1983).

In experimentation carried out in our laboratory, we were able to replicate the choose-short effect of Spetch and Wilkie $(1982,1983)$. Four pigeons were trained to peck one comparison stimulus after a 2-sec duration of sample light illumination and another comparison stimulus after a 10-sec duration of the sample light. When tested for retention at a 0 -sec delay, pigeons were $89 \%$ accurate after the long stimulus and $85 \%$ accurate after the short stimulus. At a 5-sec delay, their accuracy fell to $53 \%$ for the long sample and $61 \%$ for the short sample. Finally, at a 20 -sec delay, pigeons made only $34 \%$ correct responses to the long sample but made $70 \%$ correct responses to the short sample stimulus. Just as Spetch and Wilkie found, choice of the short-sample key dominated responding at longer delays.

As a means of providing differential experimental predictions from coding and subjective-shortening models, our pigeons were trained to respond to a third comparison stimulus. In addition to the 2- and 10-sec sample stimuli, 0-sec sample-stimulus trials were introduced. Pigeons were rewarded for pecking a red comparison key after the short, 2-sec sample and a green comparison key after the long, 10-sec sample; on 0-sec sample-stimulus trials, the comparison stimuli appeared with no prior illumination of the sample light and the rewarded response was a peck on a white comparison key. When pigeons were performing this discrimination at a high level of accuracy at a 0 -sec delay, testing was carried out over a number of delay intervals.

The subjective-shortening model and the coding model appear to make clearly different predictions about the outcome of this experiment. The predictions from each model are depicted in the two panels shown in Figure 1. Each panel shows predicted shifts in the preferences for the three comparison keys as the delay increases following the presentation of a long sample stimulus. Both models suggest that choice of the long-sample key will decline over the delay, whereas choice of the 0-sec or no-sample key will increase. In the case of the subjective-shortening model, these changes should arise from the subjective

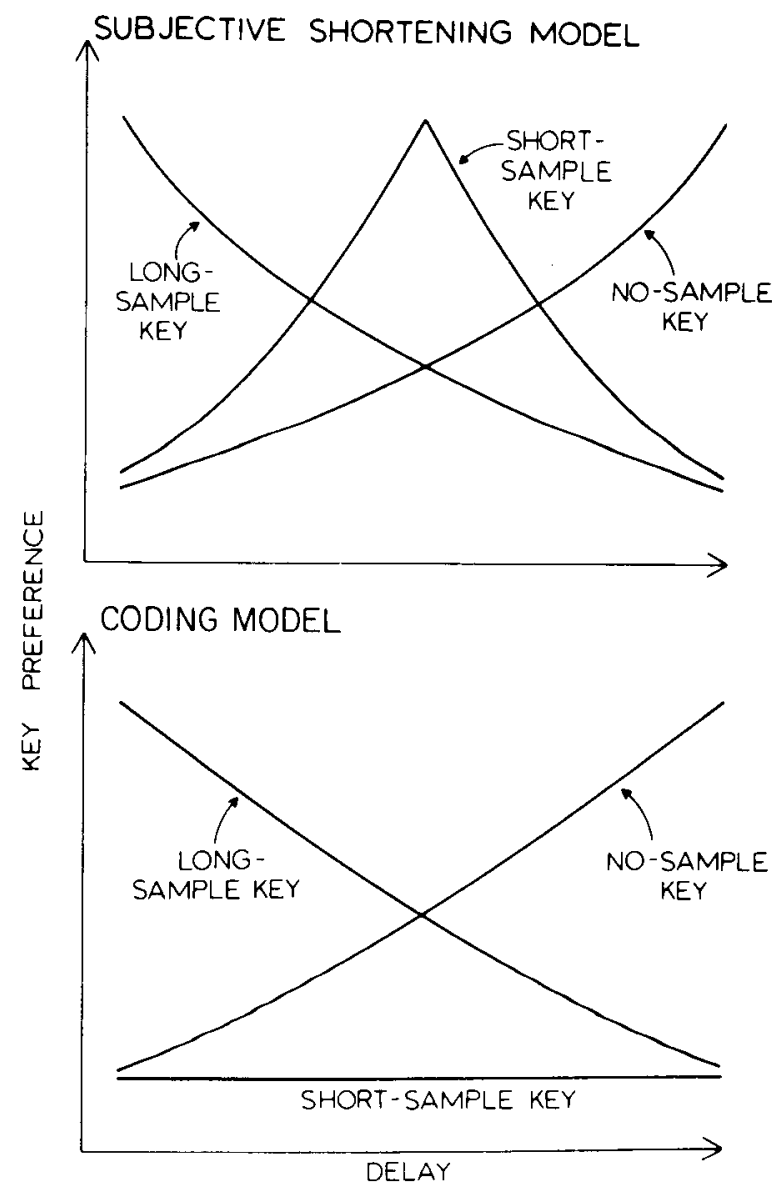

Figure 1. Predictions from a subjective-shortening model (upper panel) and a coding model (lower panel) about shifts in comparison key preference over increasing delay intervals following presentation of a long sample stimulus. 
shortening of memory of the long sample stimulus to a value at or near zero. For the recoding model, these shifts in key preference result from the increased forgetting of the long-sample-stimulus category or response instruction and a response based on the existing signal duration of $0 \mathrm{sec}$.

A clear difference between the models is seen when we examine preference for the short-sample key as a function of delay. As seen in the top panel, the subjectiveshortening model predicts that responding to the shortsample key will rise to a peak after responding to the longsample key has begun to decline and before responding to the no-sample key has reached its peak. As memory of the long sample stimulus shortens, it must pass through a value that matches the length of the short sample stimulus, and, at this point, responding to the short-sample key should be dominant. In the lower panel, by contrast, the coding model predicts no peaking of responses to the short-sample key and only a constant low level of response to this key over all delay intervals. Since only the long sample stimulus is presented on these trials, only two response instructions should control choices, the instruction to peck the long-sample key, based on categorical coding of the long sample stimulus, and, if it is forgotten, the instruction to peck the no-sample key. A low level of responding to the short-sample key may arise from either miscodings of the long sample stimulus or generalization errors to the comparison keys, but the level of responding to the short-sample key should not vary between delays.

\section{METHOD}

\section{Subjects}

Four adult Silver King pigeons served as subjects. The subjects were maintained at $80 \%$ of free-feeding weights throughout the experiment, and they had constant access to water and health grit. The birds were housed with other pigeons in a room where the temperature was kept at $22^{\circ} \mathrm{C}$. Windows in the housing room provided daylight illumination, and overhead fluorescent lights were turned on at 8:30 a.m. and turned off at 10:30 p.m. Testing was carried out between 9:00 a.m. and 1:00 p.m. for 6 days each week.

\begin{abstract}
Apparatus
All testing was conducted in a standard operant chamber for pigeons. The floor dimensions of the chamber were $31 \times 35.5 \mathrm{~cm}$, and the wall height was $35.3 \mathrm{~cm}$. The front wall of the chamber contained a row of three pecking keys, level with a pigeon's head and spaced $8 \mathrm{~cm}$ apart center to center. An electromechanical food hopper provided mixed grain through a $6 \times 6 \mathrm{~cm}$ opening centered on the front wall below the pecking keys. An amber jeweled light, containing a 24-V, 6-W lamp, was mounted on the front panel, $5 \mathrm{~cm}$ above the center pecking key. Tri-lamps were mounted behind each pecking key, and a red, green, or white comparison stimulus could be projected on any one of the three keys. All trial events and response recording were controlled by electromechanical equipment located in a room adjacent to that containing the test chamber.
\end{abstract}

\section{Procedure}

All four pigeons had previously been trained to match red and green comparison stimuli to short- and long-duration sample stimuli. For the present experiment, these birds were trained to a high level of accuracy on sessions that required response to still a third com- parison stimulus when no sample stimulus had been presented. The sample stimulus was the duration for which the amber jeweled light was illuminated. The comparison stimuli were the illumination of the three pecking keys with red, green, and white fields. Immediately after the presentation of the sample stimulus, the three comparison keys were illuminated; the configuration of lights varied quasi-randomly from trial to trial, so that each color appeared equally often at each key position. Reinforcement was delivered following a peck to the red comparison stimulus on 2-sec sample-stimulus trials and following a peck to the green key on 10-sec samplestimulus trials. A peck on an incorrect key yielded no reward and initiated the intertrial interval. Each of these sample-stimulus durations occurred on 12 randomly ordered trials within a daily session. On the remaining 24 trials of a session, the sample stimulus was not turned on and the comparison stimuli simply appeared. On these trials, only a peck on the white comparison key delivered reinforcement. Reinforcement consisted of $2 \mathrm{sec}$ of access to grain, and trials were separated by a $20-\mathrm{sec}$ intertrial interval. Both the intertrial interval and all delays were spent in darkness. Each pigeon was trained until it achieved $85 \%$ correct responses or better over a block of five sessions.

Experimental testing began after a bird had reached criterion on the basic discrimination and lasted for 35 sessions. Within a daily session, a bird was tested for 60 trials. Twenty of these trials were no-sample stimulus trials, 10 trials were 2 -sec sample-stimulus trials with a 0 -sec delay, and 10 trials were 10 -sec sample-stimulus trials with a 0-sec delay. Delayed testing was carried out on the remaining trials, with the 2-sec sample stimulus presented on 10 trials, and the 10-sec sample stimulus presented on the other 10 trials. The delay used was constant within a session but varied across sessions. The delays used were $3,6,9,12,15,18$, and $21 \mathrm{sec}$. Each delay was used once within a block of seven sessions, with the order of delays varied randomly among birds and among the five blocks of sessions. There were two orders of 60 trials in which the various types of trials occurred in random sequences, and these orders were used on alternate sessions. Over the two orders, each of the six possible configurations of the comparison stimuli on the pecking keys occurred an approximately equal number of times for each type of trial. As in training, the intertrial interval was $20 \mathrm{sec}$ and was spent in darkness.

\section{RESULTS}

The subjects learned the three-comparison-keys discrimination to a criterion of $85 \%$ correct responses over 5 successive days. The mean of the days to reach criterion was 11.75 , with Subjects P9, P14, P15, and P16 requiring $15,7,13$, and 12 days, respectively.

Performance on trials involving presentation of the long sample stimulus is shown in Figure 2. These curves present data from all five blocks of testing and are based on 50 trials at each delay for each subject. For each delay, the percentage of responses to each of the three comparison keys is shown. In the case of each bird, there is a strong preference for the long-sample key at the 0 -sec delay, followed by a decline in choice of the long-sample key to a low level by $21 \mathrm{sec}$. Correlated with decline in response to the long-sample key is a rise in choice of the no-sample key for each subject. The long-sample-key and no-sample-key curves fall and rise at relatively slow rates for Subjects P9 and P14, and hence do not cross until delays in the 15-21 sec range. By contrast, these curves change much faster for Subjects P15 and P16, and the curves cross between delays of 3-9 sec. In general, the 

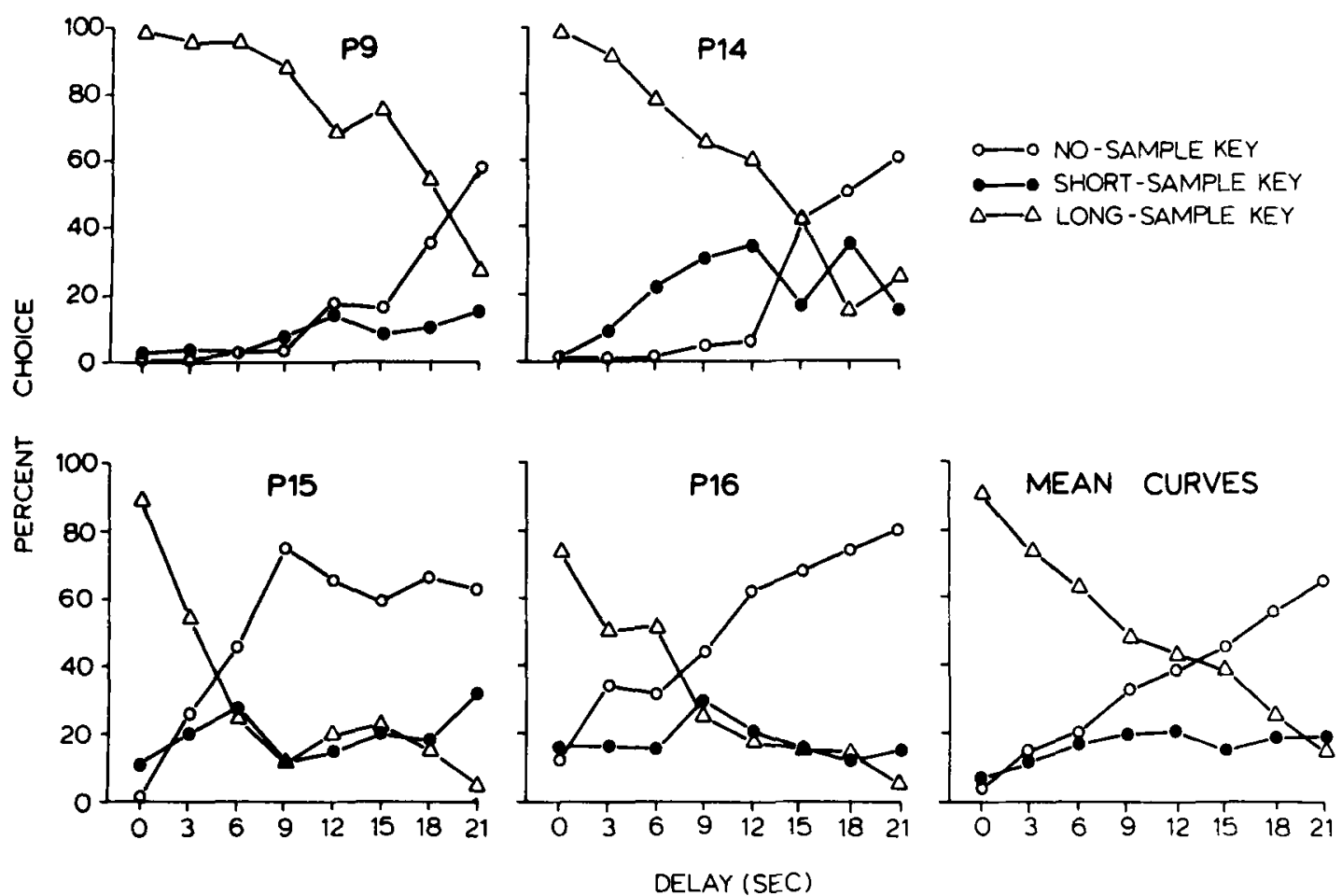

Figure 2. Curves showing the shifts in preference for comparison keys as a function of delay after presentation of a long sample stimulus.

short-sample-key curves remain low and show little variation across delays. Only the short-sample-key curve for P14 shows some degree of increase from the short delays to the longer delays. It is notable, however, that for none of the subjects is the short-sample-key curve ever higher than either the long-sample-key or the no-samplekey curve.

The changes in response preferences are particularly apparent in the mean curves. Preference for the longsample key declines monotonically as delay increases, whereas preference for the no-sample key increases monotonically. The short-sample-key curve is essentially flat. Analyses of variance performed on the mean curves showed that the drop in the long-sample-key curve was significant $[F(7,21)=17.64, p<.001]$ and that the rise in the no-sample-key curve was significant $[\mathrm{F}(7,21)=10.63$, $\mathrm{p}<.001]$. Choice of the short-sample key did not vary significantly over delay $[F(7,21)=1.50, p>.05]$.

Figure 3 shows the percentages of choices of each comparison key as a function of delay after presentation of the short sample stimulus. All four birds show an initial preference for the short-sample key that declines rapidly with increasing delay. As preference for the short-sample key weakens, choice of the no-sample key becomes dominant in each subject. The mean curves show generally monotonic falling and rising functions for the shortsample key and the no-sample key, and these curves cross at about $6 \mathrm{sec}$. The mean curve for the long-sample key appears to rise from the 0 -sec delay to a somewhat higher preference at the 3-, 6-, and 9-sec delays and then to drop to a lower level of choice at the longer delays. It is mainly Birds P9 and P14 that appear to be responsible for this variation in the long-sample-key curve. Analysis of variance indicated that the drop in the short-sample-key curve over delay was significant $[F(7,21)=14.06, p<.001]$, as was the rise in the no-sample-key curve $[F(7,21)=11.16$, $\mathrm{p}<.001]$. The variation in the long-sample-key curve across delays also was significant $[F(7,21)=2.92$, $\mathrm{p}<.05]$.

A comparison of the mean curves in Figures 2 and 3 suggests that the short-sample-key curve in Figure 3 falls faster than the long-sample-key curve in Figure 2. In support of this observation, analysis of variance showed a significant interaction between comparison key and delay $[F(7,21)=2.98, p<.01]$. Similarly, the mean curves suggest that choice of the no-sample key rises faster after a short sample stimulus than after a long sample stimulus. However, analysis of variance failed to find a significant interaction between delay and choice of the nosample key following short and long sample stimuli $(\mathrm{F}<1.00)$.

\section{DISCUSSION}

These findings appear clearly to support the coding model and to argue against the subjective-shortening model. The observation of most critical importance is the failure to find any significant variation in the short-samplekey curve throughout the delays tested. The subjective shortening model seems to require that memory of a long- 

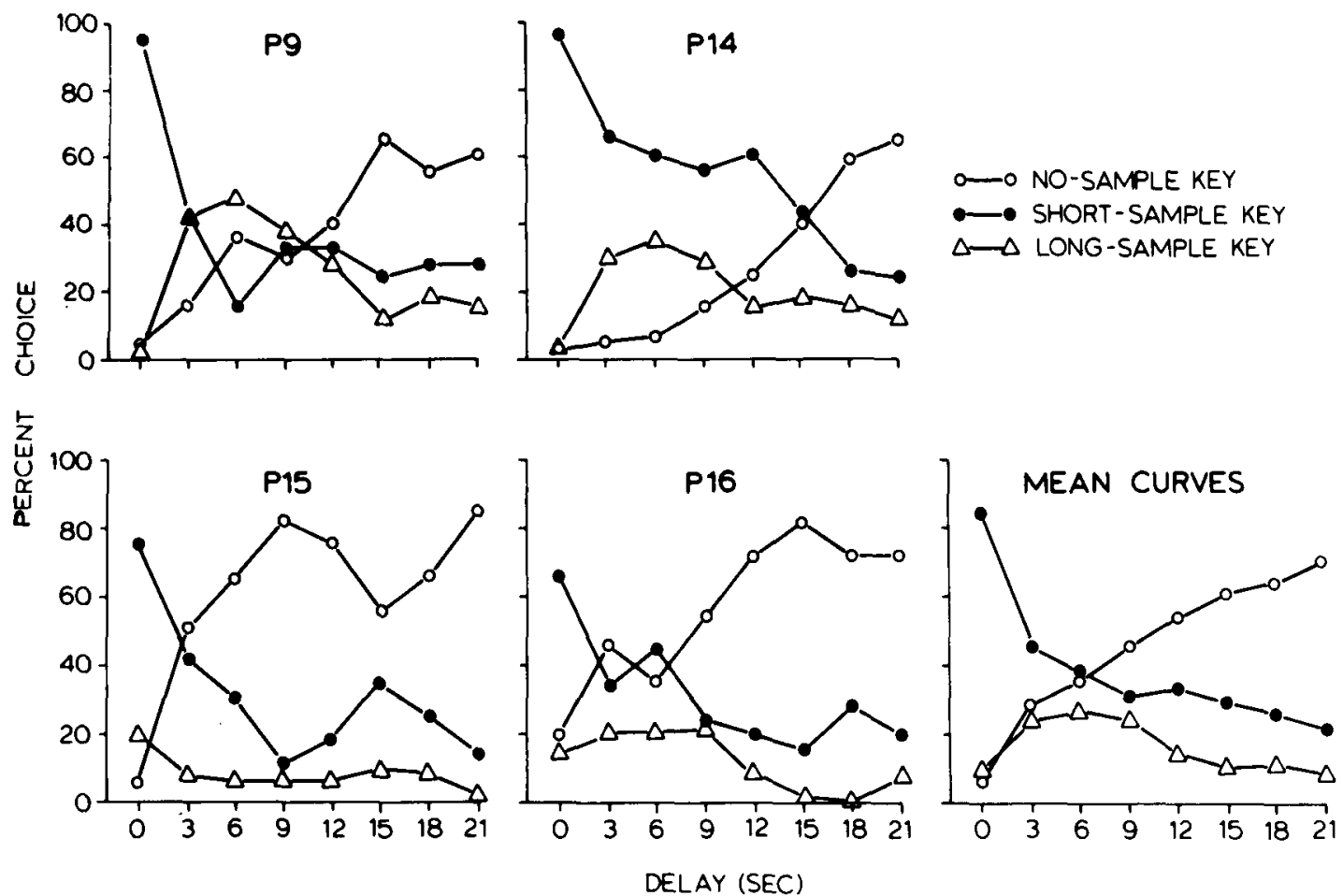

Figure 3. Curves showing the shifts in preference for comparison keys as a function of delay after presentation of a short sample stimulus.

sample stimulus must shorten to a length equivalent to the short-sample stimulus at some delay and that responses to the short-sample key should peak at this delay. The coding model, on the other hand, holds that only two codes or response instructions should control behavior after a long-sample stimulus: Animals should remember a longstimulus category or its appropriate response instruction, or, if this code is forgotten, they should respond to the key appropriate to no sample stimulus. Since the shortsample code does not arise on long-sample trials, no variation in response to the short-sample key should be found.

Two findings did appear in this experiment that are not completely consistent with either a subjective-shortening model or a coding model. One finding was the observation of a significant rise and decline over delays of the long-sample-key curve after a short sample stimulus (Figure 3). Although the effect was significant, it was marked in only two subjects, Birds P9 and P14, and should await replication before it is accepted. Similar observations have been made in animal timing experiments, and have been explained in terms of the internal clock's continuing to run during a blackout interval after a signal has been presented (Church, 1978).

A second finding that appears not to be completely consistent with a coding model is the observation that the short-sample-key curve in Figure 3 fell faster than the long-sample-key curve in Figure 2. If short and long presentations of the sample stimulus are coded into response instructions, we might expect these instructions to be forgotten at the same rates over successive delays. Yet, the data suggest slower loss of the long-sample-key instruction than of the short-sample-key instruction. In this regard, it may be worthwhile to consider how a pigeon forms a response code. When the sample light initially comes on, there is no solid basis for making a response decision. Only after the light has gone off after $2 \mathrm{sec}$ or has been on for a period of time noticeably longer than $2 \mathrm{sec}$ can the pigeon be sure the sample is short or long. There are at least three strategies a pigeon might adopt. It might initially code the sample into a peck-long instruction and change this instruction to peck short only if the sample terminates after $2 \mathrm{sec}$. The opposite possibility is that the pigeon might initially code the sample into a peckshort instruction and then change this to peck long only after the sample has been on noticeably longer than $2 \mathrm{sec}$. The third possibility is that the pigeon reserves judgment and forms a peck-short instruction only after the sample terminates at $2 \mathrm{sec}$ or a peck-long instruction after the stimulus remains on noticeably past $2 \mathrm{sec}$. In all of these cases, it can be argued that the pigeon has longer to code and rehearse the response instruction in the presence of the sample stimulus on long-sample trials than on shortsample trials. That is, simply the fact that the long sample stimulus is presented for $8 \mathrm{sec}$ longer than the short sample stimulus may give the pigeon more time to process the peck-long-sample-key instruction than the peck-shortsample-key instruction before the delay begins. If longer processing of the long-key instruction leads to greater persistence of that instruction during the delay, the difference in the rates at which long-key and short-key curves fall would be explained. Such an explanation is obviously speculative, but it seems very likely that the interaction 
of these curves may be produced by the differential initial exposure pigeons have to the short and long sample stimuli.

One reviewer has suggested that the use of a blackout period during intertrial intervals and delays in this experiment may have introduced a confound that could account for the findings shown in Figure 2. During training, nosample-stimulus trials always occurred after a 20-sec intertrial interval. It is suggested that the pigeons' choice of the no-sample white key may have come under the control of the blackout period. Since similar blackout periods were used during delays in testing, the rise in the nosample-key curve and the decline in the long-sample-key curve could be explained by an increase in the control of responding by the blackout period. It should be noted, however, that the long-sample-key curve in Figure 2 falls slowly, suggesting that control by the long-sample stimulus is relinquished gradually. If the memory of the long sample stimulus is subjectively shortening during this period, there should also be some tendency toward increased control by memory of the short sample stimulus. The fact that there is no tendency for the short-sample curve to rise throughout the $21-\mathrm{sec}$ delay period, then, still argues against subjective shortening, even given some eventual control of no-sample-key responding by the blackout during delays.

In addition to demonstrating a choose-short effect in pigeons, Spetch and Wilkie (1983) also demonstrated a choose-long effect. If pigeons were given extended training with short and long sample stimuli at a $20-\mathrm{sec}$ delay and then were shifted to a 5-sec delay, the pigeons were more accurate after long than after short samples. It was suggested that the criterion duration stored in reference memory shifts toward the shortened duration in effect at a constant delay. If the delay then is suddenly shortened, the subjective duration in working memory will be longer than the criterion duration and will yield a preference for the long-sample key. Replication of the choose-long effect has been reported recently (Spetch, 1985), and hence the effect appears to be reliable.

It could be argued, however, that the choose-long effect arises from coding processes. If pigeons are trained with short and long sample durations over repeated sessions with a long, 20-sec delay, the basis for choice between comparison stimuli may change. On the basis of the observations found in Figures 2 and 3, it may be that less persistent memories based on the short sample are lost by $20 \mathrm{sec}$, while long-sample memories are still available. The functional memory on short-sample trials then would be no sample stimulus. In this case, pigeons would need only two response rules: to peck the long-sample key when a sample stimulus had been presented and to peck the short-sample key when no sample had been presented. The distinction between short and long sample durations might be lost, and all sample presentations would be coded as "peck the long-sample key." When a pigeon then is shifted to a short 5-sec delay, the instruction to peck the long-sample key would persist for this short delay and yield a preference for the long-sample key after both short and long sample durations. With continued training at a short delay, discriminative behavior based on different codes for short and long samples would be reestablished.

This account of the choose-long effect is clearly post hoc and requires some testing, but it does suggest that the choose-long effect may be within the scope of a coding model. Furthermore, the data presented here appear to pose a problem for a subjective shortening model and offer support for the alternative coding model. It would be premature to argue that the subjective-shortening model should be replaced by a coding model, but we would argue that the coding model offers an equally plausible account of animals' memory for the duration of events.

\section{REFERENCES}

Church, R. M. (1978). The internal clock. In S. H. Hulse, H. Fowler, \& W. K. Honig (Eds.), Cognitive processes in animal behavior (pp. 277-310). Hillsdale, NJ: Erlbaum.

Church, R. M. (1980). Short-term memory for time intervals. Learning \& Motivation, 11, 208-219.

HonIG, W. K. (1978). Studies of working memory in the pigeon. In S. H. Hulse, H. Fowler, \& W. K. Honig (Eds.), Cognitive processes in animal behavior (pp. 211-248). Hillsdale, NJ: Erlbaum.

HoNig, W. K. (1981). Working memory and the temporal map. In N. E. Spear \& R. R. Miller (Eds.), Information processing in animals: Memory mechanisms (pp. 167-197). Hillsdale, NJ: Erlbaum.

HonIG, W. K., Thompson, R. K. R. (1982). Retrospective and prospective processing in animal working memory. In G. H. Bower (Ed.), The psychology of learning and motivation: Advances in research and theory (Vol. 16, pp. 239-283). New York: Academic Press.

ROBERTS, S. (1982). Cross-modal use of an internal clock. Journal of Experimental Psychology: Animal Behavior Processes, 8, 2-22.

RoberTs, W. A., \& Grant, D. S. (1976). Studies of short-term memory in the pigeon using the delayed matching-to-sample procedure. In D. L. Medin, W. A. Roberts, \& R. T. Davis (Eds.), Processes of animal memory (pp. 79-112). Hillsdale, NJ: Erlbaum.

SPETCH, M. L. (1985). Interaction between working and reference memory. Canadian Psychology, 26, 503.

SPETCH, M. L., \& WILKIE, D. M. (1982). A systematic bias in pigeons' memory for food and light duration. Behavior Analysis Letters, 2 , 267-274.

SPETCH, M. L., \&ILKIE, D. M. (1983). Subjective shortening: A model of pigeons' memory for event duration. Joumal of Experimental Psychology: Animal Behavior Processes, 9, 14-30.

(Manuscript received May 17. 1985: revision accepted for publication October 18, 1985.) 